Original Research Paper

\title{
An Investigation of Work-Related Fatigue Levels and Related Factors among Emergency Nurses: A Primary Quantitative Study
}

\author{
${ }^{1}$ Khaldoun Ismail, ${ }^{2}$ Mahmoud Al-Masaeed, ${ }^{1}$ Rawan Alsababha, \\ ${ }^{1}$ Albara Alomari and ${ }^{2}$ Muhammad Alqudah \\ ${ }^{I}$ School of Nursing and Midwifery, Western Sydney University, Australia \\ ${ }^{2}$ School of Nursing and Midwifery, Faculty of Health and Medicine, University of Newcastle, Australia
}

\author{
Article history \\ Received: $17-11-2020$ \\ Revised: 20-02-2021 \\ Accepted: 05-03-2021 \\ Corresponding Author: \\ Mahmoud Al-Masaeed \\ School of Nursing and \\ Midwifery, Faculty of Health \\ and Medicine, University of \\ Newcastle, Australia \\ Email: Mahmoud.msaeed@yahoo.com
}

\section{Introduction}

\section{Background}

Fatigue is a state of exhaustion, tiredness and loss of energy. When fatigued, an individual is unable to perform their duties and responsibilities to the optimum (Barker and Nussbaum, 2011; Al-Masaeed et al., 2020). Fatigue has an impact on reducing an individual's functioning and productivity levels. It is a phenomenon experienced across all professions. The nursing profession is not an exception to fatigue. Studies indicate prevailing fatigue levels among nurses across the globe. Previous studies have used multiple stools, including the Fatigue basement tool (FAS) and Occupational Fatigue Exhaustion Recovery tool (OFER 15), among others when measuring nurses fatigue levels (Trendall, 2000; Winwood et al., 2005; Martin, 2015; Al-Masaeed et al., 2020).

\begin{abstract}
One of the most frequent phenomena among shift workers, particularly nurses, is work-related fatigue. There is a necessary demand for research in the area concerning the relationship between socio-demographic factors, occupational variables and work-related fatigue among Arabic, fording Jordanian nurses, given the lack of knowledge. This study targets evaluating the magnitude of the relationship between socio-demographic and work in emergency care venues. A descriptive correlational study was used. A non-probability based convenient sampling technique was applied, capturing 220 emergency nurses in Jordan. Measures included socio-demographic data, Occupational variables and Fatigue Exhaustion/Recovery Scale (OFER 15). In comparison to chronic and inter-shift fatigue, acute workrelated fatigue attained the highest average score (Mean $=61.63, \mathrm{SD}$ +27.17). Acute fatigue had a significant but weak relationship with years of experience, age, marital status, income and shift-work had an imperative relationship with acute work-related fatigue. Further, the chronic fatigue and inter shift (recovery) were correlated with marital status, years of empirical data that would help develop procedures to diminish levels of work-related fatigue among Jordanian emergency nurses, which would
\end{abstract}

Keywords: Work-Related Fatigue, Chronic Fatigue, Acute Fatigue, SocioDemographic Factors, Inter-Shift Fatigue
The tools are a statistical and quantitative basis for examining the prevalence and fatigue levels among nurses. Evidence indicates that there exists fatigue among nurses. The handling of fatigue is different. While some nurses and existing systems create adaptation/mitigation strategies, others fail to employ the adaptation systems. The presence of mitigation strategies allows for adaptation and reduced fatigue to tiredness and, eventually, an adaptation to managing and controlling fatigue triggers (Sagherian et al., 2017a; 2017b). On the other hand, a failure to employ mitigation strategies leads to fatigue progression into total exhaustion and thus increased normal working conditions for the nurses. The short term implications of prolonged fatigue among nurses include health deterioration, a negative impact on their social el being 
and interactions and a decline in the nurses' motivation (Samaha et al., 2007). In the long run, the implications include long term health complications, the risk of medical errors and the increased risk of nurses exiting the nursing profession. Some of the significantly impacted nurses are the emergency department nurses. They are mandated to support, help, care for and treat critically ill and emergency cases. Such would include accident and disaster survivors, among other patient categories (LeGal et al., 2019; Al-Masaeed et al., 2020).

Unlike other hospital departments, nurses working in the Emergency Department (ED) have highly dependent patients who rely on their help for basic functions such as hygiene, including cleaning and other biological activities such as passing urine and stool. The increased demand for nurses' care and support exposes them to fatigue risk. Studies in different jurisdictions indicate that some of the prevalent types of fatigue among ED include physical, mental, acute, chronic and inter-shift recovery fatigue types (Oh et al., 2011; Ismail et al., 2019). These different fatigue types emerge due to the working conditions and the nature of expectations that the hospitals and the patients have on their nurses. Although existing literature agrees on the prevalence of fatigue among ED nurses, differences exist in prevalence levels. Some of the listed differences in the literature include (i) nurse's socio-demographics, (ii) occupational factors.

On socio-demographic factors, studies indicate a correlation between the dimensions and variables of age, gender, marital status and level of earnings on nurses' fatigue levels prevalence and their ability to address the fatigue and exhaustion risks. Equally, on occupational factors, the findings in the literature indicate a relationship between fatigue and the nurses' occupational dimensions of served departments, career ranks and functions and responsibilities on their fatigue and exhaustion levels and risks (Sawatzky and Enns, 2012; Rozo et al., 2017). Nevertheless, a preliminary analysis of the literature indicates a lack of enough literature on the factors influencing ED nurses' fatigue levels and prevalence rates in Jordan. This formed the basis for the primary study development.

\section{Aims and Objectives}

The study evaluates the levels and correlating factors of work-related fatigue among Jordanian nurses in the ED. Hence, it addresses the following research questions:

- What are the work-related fatigue levels among ED nurses in Jordan?

- Are socio-demographic factors correlated to workrelated fatigue among ED nurses in Jordan?

- Are occupational factors correlated to work-related fatigue among ED nurses in Jordan?

\section{Materials and Methods}

\section{Design, Sampling and Setting}

A descriptive correlational design was used to recruit nurses working in ED through a non-probability convenience sampling approach. The sampling size was estimated using a medium effect size of (0.22), power of (0.85) and (0.05) levels of significance, with a correlation. A sample of 179 participants was needed. The study sample size was determined based on the statistical proportion of $10 \%$ for all sample bases population base, as long as the sample base does not exceed 1000. The target ED population of nurses in Jordan was estimated at 1790 . Thus, the $10 \%$ of the sample size was at 179 respondents. The target population is ED nurses in Jordan participating hospitals. The study recruited 220 participants to compensate for dropout and any missing data. The inclusion criteria were nurses who work in ED work on a full-time basis, with work experience of at least a year before data collection time. Exclusion criteria include physical or psychological problems and pregnant female nurses. This study was carried out in the emergency department of four hospitals in Jordan, encompassing all health sectors, including governmental, private, educational and military, in different regions.

\section{Measurements}

The survey used was structured and self-administered. It consisted of the following socio-demographic questionnaire and occupational questionnaire; both are developed by the researchers based on the existing literature. In addition to that, the (OFER15) scale was used. The following paragraph discusses the scales:

1) Basic socio-demographic data questionnaire. Researchers established this scale based on existing literature and include gender, age, marital status, educational level, income and smoking

2) Occupational data questionnaire; it involves; years of experience and shift-work

3) Occupational Fatigue Exhaustion/Recovery scale (OFER15). Winwood et al. (2006a) founded the OFER15 scale. This scale consists of three subscales that assess acute fatigue, chronic fatigue and inter-shift (recovery). Each composed of five items rated on a Likert scale, using the following format, $0=$ strongly disagree to $6=$ strongly agree. Items numbers $(9,10,11,13$ and 15) were reverse coded. The total scores for each subscale ranged from 0 to 100. Each subscale was totalled up, divided by 30 and multiplied by 100 . Higher scores then indicated higher levels and a more dominant type of workrelated fatigue. This scale is valid and reliable, with a Cronbach's alpha for the total scale was $>0.84$ (Winwood et al., 2005). It was translated into 
Arabic and various other languages worldwide. In this study, the Arabic version of the OFER15 was used. The internal consistency reliability was evaluated using Cronbach's alpha, which was 0.85

\section{Ethical Considerations}

The Institutional Review Board (IRB) approval to carry out the current research was sought from the ethics committees in the Al-Zaytoonah University of Jordan (reference number 11/179/2017-2018, Ministry of Health (reference number MOH REC 180016) in addition to the ethics committee in each participated hospital. The instructions, the purposes of the study, the benefits and the risks were explained to each participant. Confidentiality and anonymity of the participants were ensured; hence, all participants' identifiers were removed from the final report. Informed consent was obtained from each participant.

\section{Data Collection}

The data was amassed from early January to late March 2018. The first researcher approached ED's in-charge nurse in every participated hospital and clarified the aim of the study. After that, the nurse in charge was asked to allow the primary researcher to contact potential participants. Those who agreed to participate and fulfilled the inclusion criteria were handed a survey. A cover letter was attached to each survey and it included a consent form, the aim of the study, a paper of instructions and an empty envelope to secure the survey completed once they complete it. Then, after one week, the researchers collected envelopes from the study volunteers.

\section{Data Analysis}

The Statistical Package for Social Sciences (SPSS), version 23.0, was used to enter and analyse data. Descriptive statistics (e.g., frequency, percentage, mean, standard deviation and range) were obtained to describe the socio-demographic characteristics and work-related fatigue variables. To research the relationship between the socio-demographic characteristics and work-related fatigue (acute, chronic and inter-shift (recovery)), correlation tests, including Pearson's and Point-biserial, were conducted. The results were deemed significant at $\mathrm{p}<0.05$. A randomised sample strategy was adopted to control the risk of confounding variables.

\section{Findings and Analysis}

\section{Socio-Demographic Characteristics of the Participants}

Out of 220 participants, $63.6 \%$ were male, $52.3 \%$ were married, $57.3 \%$ non-smokers and $82.3 \%$ reported having a bachelor's degree (Table 1). The study sample's average age was 28.54 ( $\mathrm{SD} \pm 3.78$ ), ranging between $23-46$ years. The mean of the income was 496.04 JD (SD \pm 151.16 ). The average of work experience years was 5.47 ( $\mathrm{SD} \pm 3.91)$.

\section{Occupational Factors}

All of the volunteers were working in rotating shifts with 8 hrs/day, $34.1 \%$ were on day-shifts (7 am$2 \mathrm{pm})$, while $9.5 \%$ afternoon-shifts (2-9 pm) and $19.1 \%$ were on night-shifts ( 9 pm-7 am). More details are depicted in Table 1.

\section{Levels of Work-Related Fatigue}

In Table 2, the levels of work-related fatigue are shown, including acute, chronic and inter-shift (recovery). The highest frequently reported work-related fatigue was the acute type $(\mathrm{M}=61.63$, SD +27.17), whereas, inter-shift fatigue was the least frequently reported kind of fatigue (M $=56.25, \mathrm{SD}+17.39)$. Table 2 shows the mean and the SD for all kinds of work-related fatigue.

\section{Correlation Between Socio-Demographic and Occupational Factors and Work Related Fatigue}

In Table 3, the extent of the relationship between socio-demographic variables and work related fatigue was depicted. To evaluate the direction and depth of relationship between the socio-demographic factors and work-related fatigue, Pearson (r) and Point-biserial ( $\mathrm{r}$ p.b) correlation was performed. The results showed that acute fatigue was significantly and positively related to age $(r(220)=0.18 ; \mathrm{p}<0.01)$. Income was correlated positively with acute fatigue and chronic fatigue, $(r(220)$ $=0.18 ; \mathrm{p}<0.01),(r(220)=0.33 ; \mathrm{p}<0.01)$, respectively. Years of experience was also positively correlated with acute fatigue and chronic fatigue at $(r(220)=0.39$; $\mathrm{p}<0.01),(r(220)=0.23 ; \mathrm{p}<0.01, r(220)=0.29 ; \mathrm{p}<0.01)$ respectively in addition to that, inter item shift recovery showed a significant correlation with income $(r(220)=0.24 ; \mathrm{p}<0.01)$. A critical finding in the correlation analysis was the weak correlation between the socio and occupational variables to the levels of fatigue among the ED nurses. The correlation index runs form a lowest -1 to highset 1 values. The values obtained were positive but weak, with a correlation index for relationship between marital status and inter-shift recovery fatigue as the highest of all at 0.045, which was still a weak correlation. More details are shown in Table 3. In order to study the relationship between work-related fatigue and gender, marital status, level of education, shift-work and smoking, Point-biserial correlation was performed. Table 3 demonstrates a significant positive relationship between marital status and acute fatigue $(r(220)=0.14 ; \mathrm{p}<0.05)$ and inter-shift (recovery) $(r$ $(220)=0.14 ; \mathrm{p}<0.05)$. In addition, shift-work was positively correlated with acute fatigue $(r(220)=$ $0.28 ; \mathrm{p}<0.01)$, chronic fatigue $(r(220)=0.20 ; \mathrm{p}<0.01)$ and inter-shift (recovery) $(r(220)=0.19 ; \mathrm{p}<0.01)$. 
Table 1: Socio-demographic characteristics and occupational factors of the participants $(\mathrm{N}=220)$

\begin{tabular}{|c|c|c|c|}
\hline & Characteristics & $\mathrm{N}$ & $\%$ \\
\hline \multirow{19}{*}{ Socio-demographic factors } & Age & & \\
\hline & $\mathrm{M}=28.54 ; \mathrm{SD}+3.78 ; \mathrm{R}=23-46$ years & & \\
\hline & Gender & & \\
\hline & Male & 140 & 63.6 \\
\hline & Female & 80 & 36.4 \\
\hline & Education & & \\
\hline & Diploma & 24 & 10.9 \\
\hline & Bachelor & 181 & 82.3 \\
\hline & Postgraduate & 15 & 6.8 \\
\hline & Marital status & & \\
\hline & Single & 96 & 43.6 \\
\hline & Married & 115 & 52.3 \\
\hline & Divorced & 7 & 3.2 \\
\hline & Widowed & 2 & 0.9 \\
\hline & Income & & \\
\hline & $M=496.01 ; S D+151.16 ; R=250-950$ & & \\
\hline & Smoking & & \\
\hline & Smoker & 94 & 22.7 \\
\hline & Non-smoker & 126 & 77.3 \\
\hline \multirow[t]{8}{*}{ Occupational factors } & Work-shift & & \\
\hline & Day-shift (AM) & 75 & 34.1 \\
\hline & Evening-shift (PM) & 26 & 11.8 \\
\hline & Night-shift (ND) & 42 & 19.1 \\
\hline & BC-shift & 27 & 12.3 \\
\hline & ABC-shift & 50 & 22.7 \\
\hline & Experience/years & & \\
\hline & $\mathrm{M}=5.47 ; \mathrm{SD}+3.91 ; \mathrm{R}=1-20$ years & & \\
\hline
\end{tabular}

$\mathrm{N}=$ Number, $\%=$ Percentage, $\mathrm{M}=$ Mean, $\mathrm{SD}=$ Standard Deviation, $\mathrm{R}=$ Range

Table 2: Levels of work-related fatigue among ED nurses $(\mathrm{N}=220)$

\begin{tabular}{lll}
\hline Variables & Mean & Standard deviation \\
\hline Acute fatigue & 61.63 & \pm 27.17 \\
Chronic Fatigue & 57.18 & \pm 17.41 \\
Inter-shift recovery & 56.25 & \pm 17.39 \\
\hline
\end{tabular}

Table 3: Correlation between socio-demographic and occupational factors and work-related fatigue

\begin{tabular}{|c|c|c|c|c|c|c|}
\hline \multirow[b]{3}{*}{ Factors } & \multicolumn{6}{|c|}{ Work-related fatigue } \\
\hline & \multicolumn{2}{|c|}{ Acute fatigue } & \multicolumn{2}{|c|}{ Chronic fatigue } & \multicolumn{2}{|c|}{ Inter-shift recovery } \\
\hline & $\mathrm{r}$ & $\mathrm{p}$ & $\mathrm{r}$ & $\mathrm{p}$ & $\mathrm{r}$ & $\mathrm{p}$ \\
\hline Age & 0.18 & $0.009 * *$ & 0.07 & 0.289 & 0.11 & 0.107 \\
\hline Years of experience & 0.39 & $0.000 * *$ & 0.23 & $0.000 * *$ & 0.29 & $0.000 * *$ \\
\hline \multirow[t]{2}{*}{ Income/month } & 0.41 & $0.000 * *$ & 0.33 & $0.000 * *$ & 0.24 & $0.000 * *$ \\
\hline & r p.b & $\mathrm{p}$ & r p.b & $\mathrm{p}$ & r p.b & $\mathrm{p}$ \\
\hline Gender & 0.07 & 0.339 & 0.06 & 0.368 & 0.09 & 0.200 \\
\hline Marital status & 0.14 & $0.033^{*}$ & 0.12 & 0.068 & 0.14 & $0.045^{*}$ \\
\hline Educational level & 0.11 & 0.122 & 0.13 & 0.051 & 0.05 & 0.463 \\
\hline Work-shift & 0.28 & $0.000 * *$ & 0.20 & $0.003 * *$ & 0.19 & $0.004 * *$ \\
\hline Smoking & 0.06 & 0.358 & 0.12 & 0.070 & 0.11 & 0.121 \\
\hline
\end{tabular}

*Significant at the 0.05 level; **Significant at the 0.01 level

\section{Discussion}

This research has indicated high levels of workrelated fatigue among ED nurses in Jordan; however, acute fatigue had attained the highest level. Similarly, previous studies have also reported that fatigue is prevalent among nurses, particularly the acute type of work-related fatigue (Hazzard et al., 2013; Cochran, 2014; Zhou and Fang, 2015). Possible explanations for these findings are that the duration of a work shift 
(Adriaenssens et al., 2011; Geiger-Brown et al., 2012) as well as time and the frequency of a work shift (Winwood et al., 2006b; Han et al., 2014; Dara et al., 2016) adding negative consequences on nurses' health. For example, working during night hours would alter the circadian rhythm, significantly influencing the biological o'clock (Boivin and Boudreau, 2014). The impacts of the circadian rhythm lead to erratic sleeping patterns among nurses. Consequently, the nurses are fatigued and often exhausted and even sleeping, dozing off and feeling dizzy during their working shift hours. This has the effect of increasing their in-shift acute fatigue levels. Besides, nurses in ED in Jordan undertake increased workload and responsibilities. They impact increasing their physical fatigue (due to a large number of activities per shift) and mental fatigue (due to the lack of in-shift enough breaks and relaxation exercises and techniques). Others include the existing patient culture where the co-patients increase the demand and stress on the nurses, thus not only increasing their workload but also adding to their tensions and workplace stress through numerous and, at times, uncoordinated demands by the co-patients and the patient families (Seeman, 2016; Awooda, 2020).

Consequently, the mentioned issues would increase work-related fatigue levels and lead to more stressful work conditions and vice versa. This study has shown regarding the relationship between socio-demographic factors and work-related fatigue that acute fatigue positively correlated to age, given that older nurses experienced acute fatigue more than younger nurses did. These results are coherent with previous studies that reported that aging is positively associated with workrelated fatigue among nurses (Adriaenssens et al., 2011; Meeusen et al., 2014; Rahman et al., 2017). However, this study's findings are incoherent with another previous study (Ho et al., 2013), which showed that nurses' age was negatively correlated to acute fatigue. Additionally, other previous literature supported that age and work-related fatigue were not correlated (except inter-shift (recovery) (Adriaenssens et al., 2011; Raftopoulos et al., 2012; Rahman et al., 2016). We believe that the age results are inconclusive and this could be due to different ecological locations and different genetic makeups. Hence, further studies in relation to age and work-related fatigue are paramount to reach conclusive results. This study demonstrates a positive correlation between work experience and all types of work-related fatigue. These results could relate to the fact that work capacity is reduced and work-related fatigue is increased due to working long periods in the same workplace (Hilleshein and Lautert, 2012).

Interestingly, recent studies have shown that work years' experience was negatively correlated to fatigue. Fatigue was reported more among nurses who had fewer years of experience than those who had more experience
(Ho et al., 2013; Rahman et al., 2016). This could be linked to the fact that senior nurses can cope with the constraints in relation to the time and workforce shortage. Moreover, this study revealed a positive relationship between income and work-related fatigue including, acute, chronic and inter-shift. This could be explained by the fact that low income could lead to anger, stress and demoralization for income is a basic need for the workforce to maintain good living standards (Sadeghniiat-Haghighi and Yazdi, 2015). Hence, it is plausible that those who fail to provide good income from their jobs would suffer work-related fatigue (Çelik et al., 2017). The current study results show that marital status positively correlated to acute fatigue and inter-shift (recovery) wherein married or divorced nurses reported more acute fatigue levels and inter-shift recovery than single nurses. It is well known that married nurses need to manage their work responsibilities and their marriage and home chores. The basis for the higher fatigue levels among female and married nurses could be derived from the social roles theory and culture dimensions and inclinations in Jordan.

Although a highly collective society, the social roles are strategically defined among men and women. The women in the society are allocated a larger number of domestic and home making parenting responsibilities. This adds to the list and duties on their off the shift periods. As a result, this leads to minimal rest intervals between the end of one shift and another shift. In the long run, this exposes them to increased fatigue risks than their male nursing peers (Kandolin, 1993; Oyane et al., 2013; Sagherian et al., 2017a).

Nevertheless, there lack enough quantitative findings to demonstrate the actual extent to which domestic responsibilities among married nurses increase their exposure levels to the risk of fatigue. Hence, nurses who were married experienced work-related fatigue levels that were higher in comparison to their counterparts who were single at the time of the study. The marital status for the married nurses implied additional responsibilities such as parenting and bringing up their children. Past studies have demonstrated the relationship between the nurses' number of dependants and their fatigue levels. The married nurses' dependents expanded their off the shift responsibilities scope, thus implying a low recovery rate. Their fatigue rates are predominantly higher on inter-shift recovery fatigue as contrasted to the single female nurses (Sveinsdottir, 2006; Oyane et al., 2013).

In contrast, previous studies (Raftopoulos et al., 2012; Bazazan et al., 2014) claimed no significant relationship between marital status and work-related fatigue. This study revealed a positive correlation between night work shifts or extended work shifts (BC shifts) and work-related fatigue (acute, chronic fatigue and inter-shift (recovery)). Previous studies have also 
revealed that nurses working on night-shifts reported more work-related fatigue than nurses working on day-shifts (Winwood et al., 2006b; Kunert et al., 2007). These results might be due to the negative effects a work shift has on circadian rhythm, which impacts the quality of sleep (Boivin and Boudreau, 2014), thus increasing the risk of experiencing fatigue (Kunert et al., 2007). Importantly, working on night-shifts was proven to negatively affect the workforce's health and lead to various health problems, including sleep problems, fatigue, cardiovascular and gastrointestinal diseases (Vasconcelos et al., 2012).

\section{Limitations}

The study has its main strengths in adopting and collecting primary data. It sampled and targeted Jordanian ED nurses. The results obtained were statistically analysed, presenting a quantified fatigue prevalence index and the relationship between social-demographic and occupational factors' impact on ED nurses fatigue. The OFFER 15 tool, which is validated and approved, increases the study findings' reliability. Although the study yielded significant results, the correlation was weak. This variable could have been influenced by the uneven distribution of the number of nurses for the shifts over the study development and data collection period. Further, the influences of other non-tested external factors such as teamwork and collaboration, number of nurses per shift and the management policy variances across select hospitals could have led to the weak correlation between the variables. This led to a finding where although correlated, the strength was not enough to establish a high predictor value for the independent variables influence and causative extent on the dependent factor (fatigue). The generalisation of the findings should be considered with caution. Future studies should narrow down to a specific hospital where teamwork, number of nurses per shift, policy impacts and other work environment factors can be controlled and accounted for.

\section{Practical Implications}

This study is crucial for healthcare professionals, nursing managers and policymakers in which it provides insight in relation to the safety of nurses and patients. Hence, it is vital to establish suitable measures and interventions to manage and reduce work-related fatigue levels among ED nurses. Therefore, improving the ED's work environment would directly enhance the clarity of nurses' role and their satisfaction and the nurses' and patients' outcomes. The current study results set the stage for the vitality of a continuous assessment of the work environment among nurses. Nonetheless, evaluating working conditions in light of the socio-demographic and occupational variables is vital for nurses' and patients' positive outcomes. According to their demographics and occupational factors, recognizing the needs of the emergency nurses would provide directions for development of intervention programs that specifically target work-related fatigue.

\section{Conclusion}

The findings demonstrate a correlation/relationship between fatigue levels among ED nurses and their occupation and socio-demographic variables. Nevertheless, the relationships' predictor values are weak as the interaction between the variables is not in isolation but is influenced by the external variables such as work policy, teamwork and culture. Future studies should focus on examining and controlling the extent to which the immediate environment serves as a mediating factor between fatigue and ED nurses' social-demographic and occupational factors. Further, developed policies, strategies and mitigation factors should be contextualized and customised from one ED nurse's working context and situation to the next.

\section{Acknowledgement}

I would like to express my gratitude and appreciation for supervisor in Jordan whose guidance, support and encouragement has been invaluable throughout this study. I also wish to thank the co-authors who have been a great source of help and support.

\section{Author's Contributions}

\section{Khaldoun Ismail:}

- Conceptualization

- Methodology

- Writing-Original Draft

- Visualization

Mahmoud Al-Masaeed:

- Proofreading

- $\quad$ helped write the paper

Rawan Alsababha:

- $\quad$ SPSS analysis

- Research assistant

Albara Alomari:

- $\quad$ Editing

- Proofreading

- Validation

- Supervision

Muhammad Alqudah:

- $\quad$ Proofreading 
- Validation

\section{Ethics}

Ethical concerns in the findings publication is on the privacy of the involved nurses and their site hospitals. Exposure of the nurses details risk their victimization by their respective hospital management. The findings are coded in manner that protects the identity of the nurses, thus insulating them from any potential victimization.

\section{References}

Adriaenssens, J., De Gucht, V., Van Der Doef, M., \& Maes, S. (2011). Exploring the burden of emergency care: predictors of stress-health outcomes in emergency nurses. Journal of Advanced Nursing, 67(6), 1317$1328 . \quad$ https://doi.org/10.1111/j.13652648.2010.05599.x

Al-Masaeed, M., Al-Soud, M., Alkhlaifat, E. \& Alsababha, R., (2020). An Investigation of the Impacts of Covid19 Pandemic Spread on Nurses Fatigue: An Integrated Literature Review, World Journal of Innovative Research (WJIR), Volume-9, Issue-6, pp. 20-24. https://doi.org/10.31871/WJIR.9.6.10

Awooda, E. M. (2020). Burnout Syndrome among Healthcare Workers is Associated with Violence against Them. What Needs to Be Done?. Sudan Journal of Medical Sciences, 15(2), 128-135. https://doi.org/10.18502/sjms.v15i5.7230

Barker, L. M., \& Nussbaum, M. A. (2011). Fatigue, performance and the work environment: a survey of registered nurses. Journal of Advanced Nursing, 67(6), 1370-1382. https://doi.org/10.1111/j.13652648.2010.05597.x

Bazazan, A., Rasoulzadeh, Y., Dianat, I., Safaiyan, A., Mombeini, Z., \& Shiravand, E. (2014). Demographic factors and their relation to fatigue and mental disorders in 12-hour petrochemical shift workers. Health Promotion Perspectives, 4(2), 165.

Boivin, D. B, \& Boudreau, P. (2014). Impacts of shift work on sleep and circadian rhythms. Pathology Biology, $62 \quad$ (5), 292-301. https://doi.org/10.1016/j.patbio.2014.08.001

Çelik, S., Taşdemir, N., Kurt, A., İlgezdi, E., \& Kubalas, Ö. (2017). Fatigue in intensive care nurses and related factors. The International Journal of Occupational and Environmental Medicine, 8(4), 199. https://doi.org/10.15171/ijoem.2017.1137

Cochran, K. R. (2014). A measure of perceived fatigue among nurses in western North Carolina. https://digitalcommons.gardnerwebb.edu/nursing_etd/6/?no_redirect=true
Dara, S., Tan, J., Taezoon, P. A. R. K., \& Lim, T. W. (2016). Fatigue Risk in Nurses Performing Rotating Shift Work in an Intensive Care Unit in Singapore: Analysis of Work Rosters. Singapore Nursing Journal, 43(2).

Geiger-Brown, J., Rogers, V. E., Trinkoff, A. M., Kane, R. L., Bausell, R. B. \& Scharf, S. M., (2012). Sleep, sleepiness, fatigue and performance of 12-hour-shift nurses. Chronobiology International, 29(2), pp.211219. https://doi.org/10.3109/07420528.2011.645752

Han, K., Trinkoff, A. M., \& Geiger-Brown, J. (2014). Factors associated with work-related fatigue and recovery in hospital nurses working 12-hour shifts. Workplace Health \& Safety, 62(10), 409-414. https://doi.org/10.3928/21650799-20140826-01

Hazzard, B., Johnson, K., Dordunoo, D., Klein, T., Russell, B. \& Walkowiak, P., (2013). Work-and nonwork-related factors associated with PACU nurses' fatigue. Journal of PeriAnesthesia Nursing, 28(4), pp.201-209 https://doi.org/10.1016/j.jopan.2012.06.010

Hilleshein, E. F., \& Lautert, L. (2012). Work capacity, sociodemographic and work characteristics of nurses at a university hospital. Revista latinoAmericana de Enfermagem, 20(3), 520-527. https://doi.org/10.1590/S0104-11692012000300013

Ho, J. C., Lee, M. B., Chen, R. Y., Chen, C. J., Chang, W. P., Yeh, C. Y., \& Lyu, S. Y. (2013). Work-related fatigue among medical personnel in Taiwan. Journal of the Formosan Medical Association, 112(10), 608-615. https://doi.org/10.1016/j.jfma.2013.05.009

Ismail, K. M., Malak, M. Z., \& Alamer, R. M. (2019). Psychosocial correlates of work-related fatigue among Jordanian emergency department nurses. Perspectives in Psychiatric Care, 55(3), 486-493. https://doi.org/10.1111/ppc.12354

Kandolin, I. (1993). Burnout of female and male nurses in shiftwork. Ergonomics, 36(1-3), 141-147. https://doi.org/10.1080/00140139308967865

Kunert, K., King, M. L., \& Kolkhorst, F. W. (2007). Fatigue and sleep quality in nurses. Journal of psychosocial nursing and mental health services, 45(8), 30-37. https://doi.org/10.3928/0279369520070801-07

LeGal, P., Rhéaume, A., \& Mullen, J. (2019). The long-term effects of psychological demands on chronic fatigue. Journal of Nursing Management, 27(8), 16731681. https://doi.org/10.1111/jonm.12857

Martin, D. M. (2015). Nurse fatigue and shift length: A pilot study. Nursing Economics, 33(2), 81. https://search.proquest.com/openview/1 eedef466eaff 8647c2ab4a18c7a9d2a/1?pqorigsite $=$ gscholar $\& \mathrm{cbl}=30765$ 
Meeusen, V., Hoekman, J., \& Van Zundert, A. (2014). High fatigue scores among older Dutch nurse anesthetists. AANA Journal, 82(3).

Oh, M. O., Sung, M. H., \& Kim, Y. W. (2011). Job stress, fatigue, job satisfaction and commitment to organization in emergency department nurses. Journal of Korean Clinical Nursing Research, 17(2), 215-227. https://www.koreascience.or.kr/article/JAKO20110816 4209152.page

Oyane, N. M., Pallesen, S., Moen, B. E., Åkerstedt, T., \& Bjorvatn, B. (2013). Associations between night work and anxiety, depression, insomnia, sleepiness and fatigue in a sample of Norwegian nurses. PloS One, 8(8), e70228. https://doi.org/10.1371/journal.pone.0070228

Raftopoulos, V., Charalambous, A., \& Talias, M. (2012). The factors associated with the burnout syndrome and fatigue in Cypriot nurses: a census report. BMC Public Health, 12(1), 1-13. https://doi.org/10.1186/1471-2458-12-457

Rahman, H. A., Abdul-Mumin, K., \& Naing, L. (2016). A study into psychosocial factors as predictors of work-related fatigue. British Journal of Nursing, 25(13),

$757-763$ https://doi.org/10.12968/bjon.2016.25.13.757

Rahman, H. A., Naing, L., \& Abdul-Mumin, K. (2017). Validation of the occupational fatigue exhaustion recovery (ofer) scale among emergency nurses in a brunei public hospital. The Malaysian Journal of Nursing (MJN), 8(3), 48-53. https://ejournal.lucp.net/index.php/mjn/article/vie $\mathrm{w} / 471$

Rozo, J. A., Olson, D. M., Thu, H., \& Stutzman, S. E. (2017). Situational factors associated with burnout among emergency department nurses. Workplace Health \& Safety, 65(6), 262-265. https://doi.org/10.1177/2165079917705669

Sadeghniiat-Haghighi, K., \& Yazdi, Z. (2015). Fatigue management in the workplace. Industrial Psychiatry Journal, 24(1), 12. https://doi.org/10.4103/09726748.160915

Sagherian, K., Clinton, M. E., Abu-Saad Huijer, H., \& Geiger-Brown, J. (2017a). Fatigue, work schedules and perceived performance in bedside care nurses. Workplace health \& safety, 65(7), 304-312. https://doi.org/10.1177/2165079916665398

Sagherian, K., Unick, G. J., Zhu, S., Derickson, D., Hinds, P. S., \& Geiger-Brown, J. (2017b). Acute fatigue predicts sickness absence in the workplace: A 1-year retrospective cohort study in paediatric nurses. Journal of Advanced Nursing, 73(12), 2933-2941. https://doi.org/10.1111/jan.13357
Samaha, E., Lal, S., Samaha, N., \& Wyndham, J. (2007). Psychological, lifestyle and coping contributors to chronic fatigue in shift-worker nurses. Journal of Advanced Nursing, 59(3), 221-232. https://doi.org/10.1111/j.1365-2648.2007.04338.x

Sawatzky, J. A. V., \& Enns, C. L. (2012). Exploring the key predictors of retention in emergency nurses. Journal of Nursing Management, 20(5), 696-707. https://doi.org/10.1111/j.1365-2834.2012.01355.x

Seeman, M. V. (2016). Hierarchy and Quality of Life Among Psychiatric Service Users. Journal of Psychosocial Rehabilitation and Mental Health, 3(2), 53-60. https://doi.org/10.1007/s40737-0160054-9

Sveinsdottir, H. (2006). Self-assessed quality of sleep, occupational health, working environment, illness experience and job satisfaction of female nurses working different combination of shifts. Scandinavian Journal of Caring Sciences, 20(2), 229-237. https://doi.org/10.1111/j.1471-6712.2006.00402.x

Trendall, J. (2000). Concept analysis: chronic fatigue. Journal of Advanced Nursing, 32(5), 1126-1131. https://doi.org/10.1046/j.1365-2648.2000.01583.x

Vasconcelos, S., Marqueze, E., Gonçalves, L., Lemos, L., Araújo, L., Fischer, F. M., \& Moreno, C. R. D. C. (2012). Morbidity among nursing personnel and its association with working conditions and work organization. Work, 41(Supplement 1), 3732-3737. https://doi.org/10.3233/WOR-2012-0087-3732

Winwood, P. C., Lushington, K., \& Winefield, A. H. (2006a). Further development and validation of the Occupational Fatigue Exhaustion Recovery (OFER) scale. Journal of Occupational and Environmental Medicine, 48(4), 381-389. https://doi.org/10.1097/01.jom.0000194164.14081.06

Winwood, P. C., Winefield, A. H., \& Lushington, K. (2006b). Work-related fatigue and recovery: the contribution of age, domestic responsibilities and shiftwork. Journal of Advanced Nursing, 56(4), 438-449. https://doi.org/10.1111/j.13652648.2006.04011.x

Winwood, P. C., Winefield, A. H., Dawson, D., \& Lushington, K. (2005). Development and validation of a scale to measure work-related fatigue and recovery: the Occupational Fatigue Exhaustion/Recovery Scale (OFER). Journal of Occupational and Environmental Medicine, 47(6), 594-606. https://doi.org/10.1097/01.jom.0000161740.71049.c4

Zhou, C. F., \& Fang, J. B. (2015). Correlation between job characteristics and occupational fatigue in nurses. $\mathrm{Hu} \quad \mathrm{Li} \mathrm{Za}$ Zhi, 62(5), 41. https://search.proquest.com/openview/a52b7df3f7b3 21a93607b45d288fc372/1?pqorigsite $=$ gscholar $\& \mathrm{cbl}=866377$ 